\title{
DESCENT FROM THE FORM RING AND BUCHSBAUM RINGS
}

\author{
PETER SCHENZEL
}

\section{Introduction And Main Results}

One of the major problems in commutative algebra is to recover information about a commutative ring $A$ from known properties of the form ring $G:=G_{A}(\mathfrak{q})=\oplus_{n \geq 0} \mathfrak{q}^{n} / \mathfrak{q}^{n+1}$ with respect to some ideal $\mathfrak{q}$ of $A$. There are Krull's classical results saying that $A$ is an integral domain resp. a normal domain if $G$ is an integral domain resp. a normal domain. It follows from the work [1], [2], [8] that several other properties of a homological nature, like regularity, Cohen-Macaulayness, Gorensteinness etc., descend from $G$ to $A$. In this note we want to pursue this point of view further. To this end let $Q$ denote the homogeneous ideal of $G$ generated by all the inital forms of element of $\mathfrak{q}$. For our purposes here we investigate the local cohomology modules $H_{Q}^{\bullet}(G)$ and $H_{\mathfrak{q}}^{\bullet}(A)$ of $G$ with respect to $Q$ and of $A$ with respect to $\mathfrak{q}$ resp. For their definition and basic properties see [7]. The first result concerns the descent of the finiteness from $H_{Q}^{i}(G)$ to $H_{\mathfrak{q}}^{i}(A)$.

Theorem 1.1. Let $\mathfrak{q}$ be an ideal of a commutative Noetherian ring $A$. Assume $H_{Q}^{i}(G)$ is a finitely generated graded $G$-module for all $i<t$. Then $H_{\mathfrak{q}}^{i}(A), i<t$, is a finitely generated $A$-module.

Furthermore, let $H^{i}(Q ; G)$ and $H^{i}(\mathfrak{q} ; A)$ denote the Koszul cohomology of $G$ with respect to $Q$ and of $A$ with respect to $\mathfrak{q}$ resp. Note that changing the basis yields isomorphic cohomology modules. Hence, it is not necessary to fix a basis in our notation. It is well-known, see e.g. [7], that there are canonical homomorphisms

$$
\begin{aligned}
& f_{G}^{i}: H^{i}(Q ; G) \rightarrow H_{Q}^{i}(G) \text { and } \\
& f_{A}^{i}: H^{i}(\mathfrak{q} ; A) \rightarrow H_{\mathfrak{q}}^{i}(A) .
\end{aligned}
$$

Our next result concerns the descent of the surjectivity from $f_{G}^{i}$ to the surjectivity of $f_{A}^{i}$. Note that the surjectivity of $f_{A}^{i}$ is the crucial point 
in the investigation of local Buchsbaum rings, see [13]. For a graded $G$-module $M$ let $[M]_{k}, \quad k \in \mathbb{Z}$, denote its $k$-th graded piece.

Theorem 1.2. Assume there are integers $k$ and $t$ such that

$$
\begin{aligned}
& {\left[H_{Q}^{i}(G)\right]_{n-i}=0 \text { for } n \neq k-1, k \text { and } 0 \leq i<t \text { and }} \\
& {\left[H_{Q}^{t}(G)\right]_{n-t}=0 \text { for } n>k .}
\end{aligned}
$$

Then $f_{A}^{i}$ is surjective for all $i<t$ if and only if $f_{G}^{i}$ is surjective for all $i<t$.

If $(A, \mathfrak{m})$ is a local Noetherian ring, then the Buchsbaum property does not descend from $G=G_{A}(\mathfrak{m})$ to $A$. Counterexamples are given by Steurich (University of Essen), cf. 4.3. So our result yields as a corollary, cf. 4.1, that under certain additional assumptions on $G$ the basic ring $A$ is a Buchsbaum ring if $G$ is a Buchsbaum ring.

The third result has supplementary character. It gives a sufficient condition for $f_{G}^{i}, i<t$, to be a surjective homomorphism. As shown in Example 4.4 it is not necessary.

Theorem 1.3. Let $t$ be an integer. Assume for each $0 \leq i<j<t$ and all integers $p$ and $q$ such that

$$
\left[H_{Q}^{i}(G)\right]_{p-i} \neq 0 \text { and }\left[H_{Q}^{j}(G)\right]_{q-j} \neq 0
$$

we have $p-q \neq 1$. Then the canonical homomorphism

$$
f_{G}^{i}: H^{i}(Q ; G) \rightarrow H_{Q}^{i}(G)
$$

is surjective for $i<t$, provided $Q H_{Q}^{i}(G)=0$ for $i<t$.

The proof of 1.1 and 1.2 is based on a spectral sequence technique of Serre [12], devised for passing from the form ring to the underlying ring, see 2. Another spectral sequence argument yields the proof of 1.3. The above results apply to Buchsbaum rings, for this see 4 . We refer to [15, 5.6] for an introduction and the baic results concerning spectral sequences.

The author is grateful to Rüdiger Achilles for stimulating discussions during the preliminary draft of this note several years ago.

\section{Auxiliary Spectral Sequences}

A filtration of a ring $A$ is a decreasing sequence of ideals $\left(\mathfrak{a}_{n}\right)_{n \in \mathbb{Z}}$ of $A$ such that $\mathfrak{a}_{m} \mathfrak{a}_{n} \subseteq \mathfrak{a}_{m+n}$ and $\mathfrak{a}_{0}=A$. A filtered $A$-module is an $A$-module 
$M$ with a decreasing sequence $\left(M_{n}\right)_{n \in \mathbb{Z}}$ of $A$-submodules of $M$ such that $\mathfrak{a}_{m} M_{n} \subseteq M_{m+n}$. Let $\mathfrak{a}$ be an ideal of $A$. A filtration $\left(M_{n}\right)_{n \in \mathbb{Z}}$ of $M$ is called essentially $\mathfrak{a}$-adic if $\mathfrak{a} M_{n} \subseteq M_{n+1}$ for all $n$ and $\mathfrak{a} M_{n}=M_{n+1}$ for all suffciently large $n$., By virtue of [6, (0.11.1.3)] a filtration is called exhaustive (resp. co-discrete) provided $\cap_{n \in \mathbb{Z}} M_{n}=M$ (resp. there is an integer $m$ such that $\left.M_{m}=M\right)$.

Proposition 2.1. Let $\mathfrak{q}$ be an ideal of a commutative Noetherian ring A. Then there exists a spectral sequence

$$
E_{1}^{p q}=\left[H_{Q}^{p+q}(G)\right]_{p} \Longrightarrow{ }_{p} E^{p+q}=H_{\mathfrak{q}}^{p+q}(A)
$$

whose $E_{\infty}^{p q}$-term is the $p$-th component of the graded module associated to an essentially $\mathfrak{q}$-adic filtration of $H_{\mathfrak{q}}^{p+q}(A)$.

Proof. Based on Serre's technique [12] Achilles and Avramov, see [1], constructed a spectral sequence

$$
E_{1}^{p q}=\left[\operatorname{Ext}_{G}^{p+q}(G(M), G(N))\right]_{p} \Longrightarrow_{p} E^{p+q}=\operatorname{Ext}_{A}^{p+q}(M, N),
$$

where $M, N$ are finitely generated $A$-modules with $G(M), G(N)$ their form modules with respect to $\mathfrak{q}$. Set $N=A$ and $M=A / \mathfrak{q}^{n}$. Because $G\left(A / \mathfrak{q}^{n}\right)=G / Q^{n}, n \geq 0$, the $E_{1}$-term becomes

$$
E_{1}^{p q}=\left[\operatorname{Ext}_{G}^{p+q}\left(G / Q^{n}, G\right)\right]_{p} .
$$

By virtue of the canonical homomorphism induced by

$$
A / \mathfrak{q}^{n+1} \rightarrow A / \mathfrak{q}^{n} \text { and } G / Q^{n+1} \rightarrow G / Q^{n}
$$

both sides from a direct system. According to [6, (0.11.1)] we may form its direct limit, which yields the desired spectral sequence. Note that the filtration induced by the direct limit of the spectral sequences is in general not co-discrete. But it is always exhaustive.

The above proposition is the crucial point of our investigation. It is based on Serre's technique [12], devised for passing from the tangent cone of a variety to the variety itself. For further investigations see [1]. The next proposition concerns the Koszul cohomology. Its proof follows from [12], see also [1].

Proposition 2.2. Let $\mathfrak{q}, A, Q$, and $G$ as before. Then, there is a convergent spectral sequence

$$
E_{1}^{p q}=\left[H^{p+q}(Q ; G)\right]_{p} \Longrightarrow_{p} E^{p+q}=H^{p+q}(\mathfrak{q} ; A)
$$


whose $E_{\infty}^{p q}$-term is the p-th component of the graded module associated to an essentially $\mathfrak{q}$-adic filtration of $H^{p+q}(\mathfrak{q} ; A)$.

Proof of 1.1. Because $H_{Q}^{i}(G), i<t$, is a finitely generated graded $G$-module it follows that $\left[H_{Q}^{i}(G)\right]_{n}=0$ for all $|n| \gg 0$ and $0 \leq i<t$. That is, the filtration of $H_{\mathfrak{q}}^{i}(A), i<t$, given in 2.1, is finite. Since all the $E_{\infty}^{p q}$-terms, $p+q<t$, are annihilated by $\mathfrak{q}$ we see that $H_{\mathfrak{q}}^{i}(A), i<t$, is annihilated by a power of $\mathfrak{q}$. According to [3, Lemma 3], this completes the proof.

A litte bit more is true, if we assume $H_{Q}^{i}(G), i<t$, a $G$-module of finite length.

Corollary 2.3. Suppose $H_{Q}^{i}(G), i<t$, is a G-module of finite length. Then $H_{\mathfrak{q}}^{i}(A), i<t$, is an $A$-module of finite length and

$$
L_{G}\left(H_{Q}^{i}(G)\right) \geq L_{A}\left(H_{\mathfrak{q}}^{i}(A)\right) \text { for } i<t .
$$

The proof of 2.3 is similarly to the proof of 1.1. Hence we omit it. The particular case of 2.3 for a local ring $A$ and an $\mathfrak{m}$-primary ideal has been shown in 10, (4.2)], by a completely different technique.

As a further auxiliary result we shall use the following well-known spectral sequence, see e.g. [11, Section 2].

Proposition 2.4. There is a convergent spectral sequence

$$
E_{2}^{p q}=H^{p}\left(Q ; H_{Q}^{q}(G)\right) \Longrightarrow E^{p+q}=H^{p+q}(Q ; G)
$$

for computing the Koszul cohomology.

\section{Proof of (1.2) AND (1.3)}

Proof of 1.2. Firstly, we consider the spectral sequence given in 2.1. We claim that $E_{1}^{p q}=E_{\infty}^{p q}$ for $p+q<t$. In order to show this we consider the subsequent stages, i.e.

$$
E_{r}^{p-r, q+r-1} \rightarrow E_{r}^{p q} \rightarrow E_{r}^{p+r, q-r+1} .
$$

Assume $E_{r}^{p q} \neq 0$ for some $p+q=i<t$. Then $E_{1}^{p q} \neq 0$, i.e. $p=k-i-1$ or $p=k-i$ by virtue of the assumption. Now $E_{r}^{p+r, q-r+1}$ is a subquotient of $E_{1}^{p+r, q-r+1}=\left[H_{Q}^{i+1}(G)\right]_{p+r}=0$ for $p=k-i-1$ or $p=k-i$. Therefore, if $E_{r}^{p q} \neq 0$ we have $E_{r}^{p+r, q-r+1}=0$. The same arguments yield $E_{r}^{p-r, q+r-1}=0$ if $E_{r}^{p q} \neq 0$. That is, $E_{1}^{p q}=E_{\infty}^{p q}$ for all $p+q=i<t$. Thus, 
$H_{\mathfrak{q}}^{i}(A), \quad i<t$, possesses a filtration of two terms. Hence, there is a short exact sequence

$$
0 \rightarrow\left[H_{Q}^{i}(G)\right]_{k-i} \rightarrow H_{\mathfrak{q}}^{i}(A) \rightarrow\left[H_{Q}^{i}(G)\right]_{k-i-1} \rightarrow 0 .
$$

Secondly, we use the spectral sequence given in 2.4 in order to show that

$$
\begin{aligned}
& {\left[H^{i}(Q ; G)\right]_{n-i}=0 \text { for } n \neq k-1, k \text { and } 0 \leq i<t \text { and }} \\
& {\left[H^{t}(Q ; G)\right]_{n-t}=0 \text { for } n>k .}
\end{aligned}
$$

To this end we note that $E_{2}^{p q}=H^{p}\left(Q ; H_{Q}^{q}(G)\right)$ is by definition a subquotient of $H_{Q}^{q}(G)(p)^{\left(\begin{array}{c}m \\ p\end{array}\right)}$, where $m$ denotes the number of generators of $Q$. Therefore, $E_{2}^{p q}$ vanishes for $p+q=i<t$ (resp. $p+q=t$ ) in all graded pieces $\neq k-i-1, k-i$ (resp. $>k-t$ ). Hence, the spectral sequence proves the claim. Thirdly, we apply this result in order to investigate the spectral sequence given in 2.2. Similarly as in the first part of the proof it yields a short exact sequence

$$
0 \rightarrow\left[H^{i}(Q ; G)\right]_{k-i} \rightarrow H^{i}(\mathfrak{q} ; A) \rightarrow\left[H^{i}(Q ; G)\right]_{k-i-1} \rightarrow 0
$$

for $i<t$. Therefore, the canonical homomorphisms $f_{G}^{i}$ and $f_{A}^{i}$ induce a commutative diagram with exact rows

$$
\begin{aligned}
& 0 \rightarrow\left[H^{i}(Q ; G)\right]_{k-i} \rightarrow H^{i}(\mathfrak{q} ; A) \rightarrow\left[H^{i}(Q ; G)\right]_{k-i-1} \rightarrow 0 \\
& \downarrow\left[f_{G}^{i}\right]_{k-i} \quad \downarrow f_{A}^{i} \quad \downarrow\left[f_{G}^{i}\right]_{k-i-1} \\
& 0 \rightarrow\left[H_{Q}^{i}(G)\right]_{k-i} \quad \rightarrow \quad H_{\mathfrak{q}}^{i}(A) \quad \rightarrow \quad\left[H_{G}^{i}(G)\right]_{k-i-1} \quad \rightarrow \quad 0
\end{aligned}
$$

for $i<t$, where $\left[f_{G}^{i}\right]_{n}$ denotes the $n$-th graded piece of $f_{G}^{i}$. According to $3.1\left[f_{G}^{i}\right]_{k-i}$ is always surjective. Hence, the snake lemma yields that $f_{A}^{i}$, $i<t$, is surjective if and only if $f_{G}^{i}, i<t$, is ssurjective.

Proposition 3.1. Suppose there exists an integer $k$ such that

$$
\left[H_{Q}^{i}(G)\right]_{k+1-i}=0 \text { and all } i \in \mathbb{Z} \text {. }
$$

Then the canonical homomorphism

$$
\left[f_{G}^{i}\right]_{k-i}:\left[H^{i}(Q ; G)\right]_{k-i} \rightarrow\left[H_{Q}^{i}(G)\right]_{k-i}
$$

is surjective for all $i$.

Proof. For this we have to investigate the spectral sequence given in 2.4. We claim $\left[E_{2}^{0 i}\right]_{k-i}=\left[E_{\infty}^{0 i}\right]_{k-i}$ for all $i$. To this end we are looking at the 
subsequent stages. Incoming d's come from subquotients of $E_{2}^{-r, i+r-1}=$ 0 . Outgoing $d$ 's land in subquotients of

$$
\left[E_{2}^{r, i-r+1}\right]_{k-i}=\left[H^{r}\left(Q ; H_{Q}^{i-r+1}(G)\right)\right]_{k-i}
$$

which is a subquotient of $\left[H_{Q}^{i-r+1}(G)(r)\left(\begin{array}{c}m \\ r\end{array}\right)\right]_{k-i}=0$, by virtue of the assumption. Here $m$ denotes the number of generators of $Q$. Hence the claim is proved. Next we note that

$$
\left[E_{2}^{0 i}\right]_{k-i}=\left[H^{0}\left(Q ; H_{Q}^{i}(G)\right)\right]_{k-i} \simeq\left[H_{Q}^{i}(G)\right]_{k-i}
$$

because $\left[H_{Q}^{i}(G)\right]_{n-i}=0$ for $n>k$ by the assumption. But now the module $\left[H^{i}(Q ; G)\right]_{k-i}$ possesses a filtration whose associated 0 -th graded piece is $\left[E_{\infty}^{0 i}\right]_{k-i}$, i.e. there is a canonical surjective mapping

$$
\left[H^{i}(Q ; G)\right]_{k-i} \rightarrow\left[H_{Q}^{i}(G)\right]_{k-i}
$$

for all $i$. By virtue of the functoriality of the spectral sequence it is noting else but $\left[f_{G}^{i}\right]_{k-i}$.

Proof of 1.3. We use the spectral sequence

$$
E_{2}^{p q}=H^{p}\left(Q ; H_{Q}^{q}(G)\right) \Longrightarrow E^{p+q}(Q ; G)
$$

given in 2.4. Because of $Q H_{Q}^{i}(G)=0, i<t$, it follows that

$$
E_{2}^{p q}=H_{Q}^{q}(G)(p)^{\left(\begin{array}{c}
m \\
p
\end{array}\right)} \text { for } p+q<t .
$$

Here $m$ denotes the number of generators of $Q$. In the subsequent stages we have

$$
E_{r}^{p-r, q+r-1} \rightarrow E_{r}^{p q} \rightarrow E_{r}^{p+r, q-r+1}
$$

Suppose that

$$
\left[E_{r}^{p q}\right]_{n} \neq 0 \text { for some } p+q<t,
$$

then $\left[E_{2}^{p q}\right]_{n} \neq 0$. Because $E_{r}^{p+r, q-r+1}$ resp. $E_{r}^{p-r, q+r-1}$ is derived from $H^{p+r}\left(Q ; H_{Q}^{q-r+1}(G)\right)$ resp. $H^{p-r}\left(Q ; H_{Q}^{q+r-1}(G)\right)$ it follows that

$$
\left[E_{r}^{p+r, q-r+1}\right]_{n}=0 \text { resp. }\left[E_{r}^{p-r, q+r-1}\right]_{n}=0
$$

by virtue of the assumption. That is, $E_{2}^{p q}=E_{\infty}^{p q}$ for $p+q<t$. Because $H^{i}(Q ; G)$ possesses a filtration whose associated $i$-th graded piece is $E_{\infty}^{0 i}$ there is a canonical surjective homomorphism

$$
H^{i}(Q ; G) \rightarrow H_{Q}^{i}(G), \quad i<t .
$$


By virtue of the functoriality of the considered spectral sequence it is nothing else but $f_{G}^{i}$, i.e. $f_{G}^{i}, i<t$, is surjective, as required.

\section{Applications to Buchsbaum Rings}

In this section $(A, \mathfrak{m})$ denotes a local Noetherian ring of dimension $d$ with $\mathfrak{m}$ its maximal ideal. Then $A$ is called a Buchsbaum ring if the difference $C(A):=L(A / \mathfrak{q})-e(\mathfrak{q} ; A)$ is an invariant of $A$ not depending on the choice of a parameter ideal $\mathfrak{q}$ of $A$. Here $L(A / \mathfrak{q})$ and $e(\mathfrak{q} ; A)$ denote resp. the length of $A / \mathfrak{q}$ and the multiplicity of $A$ with respect to $\mathfrak{q}$, see [14 for further details. In his crucial paper 113 Stückrad showed that $A$ is a Buchsbaum ring if and only if the canonical homomorphism $f_{A}^{i}: H^{i}(\mathfrak{m} ; A) \rightarrow H_{\mathfrak{m}}^{i}(A), i<d$, is surjective. Let $G=G_{A}(\mathfrak{m})$ denote the associated form ring and $M=G_{+}$the irrelevant maximal ideal of $G$. The graded ring $G$ is called a Buchsbaum ring if $G_{M}$ is a local Buchsbaum ring. Hence, our main results 1.2 and 1.3 apply to the situation of Buchsbaum rings.

Corollary 4.1. Let $G=G_{A}(\mathfrak{m})$ denote the associated graded form ring. Assume there is an integer $k$ such that

$$
\begin{aligned}
& {\left[H_{M}^{i}(G)\right]_{n-i}=0 \quad \text { for } \quad n \neq k-1, k \text { and } 0 \leq i<d \quad \text { and }} \\
& {\left[H_{M}^{d}(G)\right]_{n-d}=0 \quad \text { for } n>k \text {. }}
\end{aligned}
$$

Then $A$ is a Buchsbaum ring if and only if $G$ is a Buchsbaum ring. In this case $L\left(H_{M}^{i}(G)\right)=L\left(H_{\mathfrak{m}}^{i}(A)\right)$ for all $0 \leq i<d$.

Proof. Readily it follows from 1.2 using the characterization of Buchsbaum rings in terms of the surjectivity of $f_{A}^{i}$ and $f_{G}^{i}$. The statement on the length of the local cohomology modules is clear by virtue of the short exact sequence given in the proof of 1.2 .

The 'only if' part of 4.1 is one of the main results of Goto's paper [5, Theorem (1.1)]. His proof is completely different. It does not use Serre's spectral sequence technique for passing from the tangent cone to the ring.

Corollary 4.2. Let $G$ be as in 4.1 . Assume for each $0 \leq i<j<d$ and all integers $p$ and $q$ with

$$
\left[H_{M}^{i}(G)\right]_{p-i} \neq 0 \text { and }\left[H_{M}^{j}(G)\right]_{q-j} \neq 0
$$

we have that $p-q \neq 1$. Then $G$ is a Buchsbaum ring, provided $M H_{M}^{i}(G)=0$ for $i<d$. 
The proof follows by virtue of 1.3 accordingly to the fact that $G$ is a Buchsbaum ring if $f_{G}^{i}, i<d$, is surjective. Note that 4.2 was shown independently by Stückrad [14, Prop. 3.10] not using a spectral sequence technique. Particular cases of it were obtained in [9], resp. by Goto and others.

We conclude this section with two examples concerning the assumptions in 1.2 and 1.3. The following example was given by Steurich, see also [5, (4.10)].

Example 4.3. The condition in 1.2 is the best possible. Set

$$
A=k[|x, y, z|] /\left(x^{2}, x y, x z-y^{r}, y^{r+1}, x z^{2}\right), r \geq 3 \text { an integer, }
$$

where $k[|x, y, z|]$ denotes the formal power series ring over a field $k$. Note that $\operatorname{dim} A=1$. Then

$$
G:=G_{A}(\mathfrak{m})=k[x, y, z] /\left(x^{2}, x y, x z, y^{r+1}, y^{r} z\right)
$$

is a Buchsbaum ring, i.e. $f_{G}^{0}$ is surjective. Furthermore,

$$
\begin{aligned}
H_{M}^{0}(G) & =G / M(-1) \oplus G / M(-r), \\
{\left[H_{M}^{1}(G)\right]_{n} } & =0 \text { for } n>r-2 \text { and }\left[H_{M}^{1}(G)\right]_{r-2} \neq 0 .
\end{aligned}
$$

On the other hand, $A$ is not a Buchsbaum ring, i.e. $f_{A}^{0}$ is not surjective.

While the finite length of $H_{M}^{i}(G), i \neq \operatorname{dim} G$, is inherited to $H_{\mathfrak{m}}^{i}(A), i \neq$ $\operatorname{dim} A$, for the form ring $G=G_{\mathfrak{m}}(A)$ of a local $\operatorname{ring}(A, \mathfrak{m})$, see 1.1, this is not true in the Buchsbaum case. It does not hold even in the quasiBuchsbaum case, where quasi-Buchsbaum means that for $i \neq \operatorname{dim} A$ the local cohomology is a vector space over $A / \mathfrak{m}$. This follows because the local ring $(A, \mathfrak{m})$ in 4.3 is not quasi-Buchsbaum. So one might continue in order to improve the result in 1.1 by taking into acount the more subtle behaviour of $k$-Buchsbaum rings.

The next example shows that the assumption in 1.3 is not necessary for $f_{G}, i<t$, surjective.

Example 4.4. Let $R=k\left[x_{1}, \ldots, x_{6}\right]$ denote the polynomial ring over an infinite field $k$. Using a technique of Griffith and Evans in [4] Goto constructed examples of homogeneous prime ideals $P \subset R$ such that $R_{1}:=R / P$ is a 4 -dimensional graded domain with

$$
\begin{aligned}
H_{M}^{i}\left(R_{1}\right) & =0, \quad i \neq 1,4, \quad H_{M}^{1}\left(R_{1}\right) \simeq k(-2), \quad \text { and } \\
{\left[H_{M}^{4}\left(R_{1}\right)\right]_{n} } & =0 \text { for all } n \geq 0 .
\end{aligned}
$$


Let $R_{2}=k\left[y_{1}, y_{2}, y_{3}\right] / F, F$ a homogeneous form of degree 3 , be the coordinate ring of a plane cubic. Note that $R_{2}$ is a two-dimensional Cohen-Macaulay ring with $\left[H_{M}^{2}\left(R_{2}\right)\right]_{n}=0$ for all $n \geq 1$ and $\left[H_{M}^{2}\left(R_{2}\right)\right]_{0}=$ $k$. Let $S$ denote the Segre product of $R_{1}$ and $R_{2}$ over $k$. Using the Künneth formula, as done in [9, Section 5], we obtain

$$
\begin{aligned}
& H_{M}^{i}(S)=0, \quad i \neq 1,2,5, \quad H_{M}^{1}(S) \simeq k^{10}(-2), \quad \text { and } \\
& H_{M}^{2}(S) \simeq k(0) .
\end{aligned}
$$

Therefore, the assumptions of 1.3 are not fulfilled because in this case $(1+2)-(2+0)=1$. We show that $f_{S}^{i}, i<5$, is surjective. For $i \neq 0,3,4$ this is trivially true. Using 2.4 it follows readily for $i=1$. Therefore, it is enough to show the surjectivity of $\left[f_{S}^{2}\right]_{0}$. The Künneth relations induce a commutative diagram

$$
\begin{aligned}
{\left[R_{1}\right]_{0} \otimes\left[H^{2}\left(M ; R_{2}\right)\right]_{0} } & \rightarrow\left[H^{2}(M ; S)\right]_{0} \\
\downarrow[\mathrm{id}]_{0} \otimes\left[f_{R_{2}}^{2}\right]_{0} & \downarrow\left[f_{S}^{2}\right]_{0} \\
{\left[R_{1}\right]_{0} \otimes\left[H_{M}^{2}\left(R_{2}\right)\right]_{0} } & \simeq\left[H_{M}^{2}(S)\right]_{0} .
\end{aligned}
$$

Because $R_{2}$ is a Cohen-Macaulay ring with $\left[H_{M}^{2}\left(R_{2}\right)\right]_{n}=0$ for all $n \geq 1$ it follows easily that $\left[f_{R_{2}}^{2}\right]_{0}:\left[H^{2}\left(M ; R_{2}\right)\right]_{0} \rightarrow\left[H_{M}^{2}\left(R_{2}\right)\right]_{0}$ is an isomorphism. Hence, $\left[f_{S}^{2}\right]_{0}$ is surjective as required.

\section{REFERENCES}

[1] R. Achilles, L. Avramov: Relations between properties of a ring and of its associated graded ring, Sem. Eisenbud, Singh, Vogel, Vol. II, Teubner Texte zur Mathematik 48 (1982), 5-29.

[2] M.P. Cavalieri, G. Niesi: On Serre's condition in the form ring of an ideal, J. Math. Kyoto Univ. 21 (1981), 537-546.

[3] G. Faltings: Über die Annullatoren lokaler Kohomologiegruppen, Archiv der Math. 30 (1978), 473-476.

[4] S. Goto: Rings with linear resolution, In: Proc. of the 9th Intern. Symp. of the Tanaguchi Foundation, Conf. on Comm. Algebra, Katata, 1981, pp. 4-11.

[5] S. Goto: Noetherian local rings with Buchsbaum associated graded rings, J. of Algebra 86 (1984), 336-384.

[6] A. Grothendick: Elements de geometrie algebrique, $I I I_{1}$, Publ. Math., I.H.E.S. 11 (1961).

[7] A. Grothendieck: Local cohomology, Notes by R. Hartshorne, Lecture Notes in Math. 41 (1967).

[8] M. Hochster, L.J. Ratliff, JR.: Five theorems on Macaulay rings, Pacific J. Math. 44 (1973), 147-172. 
[9] P. Schenzel: Applications of dualizing complexes to Buchsbaum rings, Advances in Math. 44 (1982), 61-77.

[10] P. SChenzeL: Standard systems of parameters and their blowing-up rings, J. Reine Angew. Mathematik 344 (1983), 201-220.

[11] P. Schenzel: Applications of Koszul homlogy to numbers of generators and syzygies, J. Pure and Appl. Alg., to appear.

[12] J.-P. Serre: Algèbre locale - Multiplicités, Lecture Notes in Math. 11 (1965).

[13] J. StüCKRAD: Über die kohomologische Charakterisierung von BuchsbaumModuln, Math. Nachr. 95 (1980), 265-272.

[14] J. Stǘckrad, W. Vogel: 'Buchsbaum Rings and Applications', VEB Deutscher Verlag der Wiss., Berlin, 1986.

[15] C. Weibel: 'An Introduction to Homological Algebra', Cambr. Univ. Press, 1994.

Martin-Luther-Universität Halle-Wittenberg, Fachbereich Mathematik und Informatik, D - 06099 Halle (SaAle), Germany

E-mail address: schenzel@mathematik.uni-halle.de 